\title{
SOURCES OF GRAVITATIONAL RADIATION: COALESCING BINARIES
}

\author{
B. F. Schutz \\ Department of Physics, University of Wales College of Cardiff, P.O. Box 78, \\ Cardiff CF1 IXL, U.K.
}

ABSTRACT

Binaries consisting of compact objects -- neutron stars, or black holes -- seem likely to become the source of gravitational radiation most frequently observed in the late 1990 's by the ground-based, laser-interferometric detectors now under development. The radiation comes from the orbital motion as the stars sptral together due to gravitational radiation reaction. The low frequencies observable from space make new classes of binarles detectable, such as coalescing massive black holes, and make it possible that several coalescencing systems with relatively long times to coalescence could be detected in our own galaxy. I examine the prospects for detecting such sources with various types of space-based detectors, and then consider the sorts of things we could learn from them, including new tests of general relativity.

\section{INTRODUCTION TO COALESCING BINARIES}

There are many classes of binary systems that emit detectable gravitational radiation in the low frequency regime sulted to observations from space. (See Thorne /1/ for a recent review.) The class that is perhaps the hardest to predict, and which therefore could be the most rewarding to observe, is the coalescing binary. A good working definition of a coalescing binary is a system whose components are massive enough and close enough together for the loss of energy and angular momentum in gravitational waves to cause a significant evolution of the system during the period of observation. These systems were first considered as potential sources of gravitational radiation by Clark \& Eardley 121

Our definition requires that the components be compact objects: white dwarfs, neutron stars, or black holes. Binaries consisting of main sequence stars are not relativistic enough to have a sufficiently short gravitational radiation evolution timescale. During all but the very late stages of the evolution of systems containing neutron stars and/or black holes, the orbital motion can be very accurately modelled as a Newtonian point-mass binery, with gravitational radiation reaction as given by the 'quadrupole formula' $/ 3 \%$. In most circumstances it is also permissible to take the orbital eccentricity of the model equal to zero, since gravitational radiation reduces the eccentricity faster than it does the orbital radius. This model gives the following basic equations describing the gravitational waves from a system consisting of two objects with total mass $M \times M_{0}$ and reduced mass $\mu \times M_{0}$ at a distance $r \times 10 \mathrm{kpc}$, whose quadrupole radiation comes of $f$ at the frequency $f \times$ $0.1 \mathrm{~Hz}$. For the amplitude we have

$$
h_{\max }=2.59 \times 10^{-21} \mu M^{2 / 3} f^{2 / 3} r^{-1}
$$

By $h_{\text {max }}$ we mean the largest amplitude when the system and the detector are most favourably oriented with respect to each other. The typical r.m.s. amplitude, averaged over orientations of both the binary system and the detector, is somewhat smaller: for an interferometric detector it is down by 15. The timescale for decay of the orbit is given by

$$
\tau=\mathrm{f} / \dot{f}=7.97 \times 10^{8} \mu^{-1} \mathrm{M}^{-2 / 3} \mathrm{f}-8 / 3 \text { sec. }
$$

Because the orbital decay accelerates so quickly, the actual lifetime is $3 / 8$ of this timescale Notice that, since both $h$ and $\tau$ are measureable, one can take their product to find $r$ : the masses $M$ and $\mu$ drop out. This ability to measure $r$ is almost unique in astronomy, and it forms the basis of a new method of determining Hubble's constant from ground-based gravitational wave observations at kilotertz frequencies $14,5 \%$. However, it is important to note that $h$ itself can only be measured it 
the varfous orientation angles can be determined, and this normally requires 3 or 4 detectors to provide enough information. If space-based detections involve, say, only a single interferometer, then only an estimate of $h$ and therefore of $r$ will be possible.

It is clear from this that with reasonable sensitivity one could expect to detect any systems consisting of white dwarfs, neutron stars or solar-moss black holes if they are in our galaxy, and that they will be changing in the observation time if they have a frequency higher than $0.1 \mathrm{~Hz}$ or so. By rescaling the same equations to larger and more distant masses, we can see that we would get a similar amplitude (larger by a factor of about 1.71) and a similar timescale (smaller by the same factor) for a system consisting of two $10^{*} \mathrm{M}_{0}$ black holes at a distance of 10 Gpc (cosmological redshift considerably larger than 1) radiating at a frequency of $10^{-*} \mathrm{~Hz}$. (This ignores cosmological redshift and curvature effects, to which I will return below.)

The signal will only stand out from the noise over a long integration time. If the system is not chenging in frequency significantly over the period of observation, then simple Fourier analysis will pick it out, provided that there is no confusion with other binary systems. The signal-to-nolse ratio w1ll be proportional to the square root of the observing period. If the frequency does change significantly, then matched filtering of the data will be necessary, and the signal-to-nolse ratio will improve by the square root of $\tau$. In principle this filtering can be done in the same way as is being planned for ground-based detection: see the articles in reference $/ 6 /$. It is possible that the background 'noise' of other binary systems will be reduced by a larger factor by matched filtering than would true white noise sources, such as shot notse in an interferometer, since the binary signals are phase-coherent over long periods of time, and not necessarily very densely spaced in frequency.

\section{KEY NUMBERS FOR COALESCING BINARIES}

The formulas in the last section help us get a rough idea of the sorts of systems we can observe. There are a few useful numbers that discriminate among important regimes of detection.

Given an observing time $T \times 10^{7} \mathrm{sec}$, what is the frequency $f_{\text {conlasing }}$ of a system whose remaining lifetime equals $T$ ? This is a system that can be followed all the way to coalescence in the time of observation, using of course ground-based detectors if necessary for the higher frequencles in the late stages of coalescence. The critical frequency 1s:

$$
f_{\text {coalescing }}=0.52 \mu^{-3 / 8} \mathrm{M}^{-1 / 4} \mathrm{~T}^{-3 / 8} \mathrm{~Hz} \text {. }
$$

For Galactic systems, this frequency is rather too high to be accessible to many space-based detectors. If we were generous and imagined that a space-based detector could identify a system that might coalesce in a much longer time, say 20 years, which could then be picked up by groundbased systems, what would the change in the critical frequency be? The weak dependence on $T$ means that it only decreases to $0.11 \mathrm{~Hz}$, st1ll too high for presently planned detectors. For the cosmological system considered above, however, the situation is brighter: the critical frequency for two $10^{*} \mathrm{M}$ black holes is $4.2 \times 10^{-4} \mathrm{~Hz}$, a very accessible frequency.

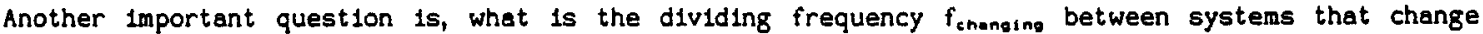
their frequency measureably in the observation time $T \times 10^{\gamma}$ sec and those that do not? The key point is that as $T$ increases, one's frequency resolution also increases, so that one can distinguish frequencles separated by as little as $1 / T$. The critical frequency is, in the same notation,

$$
f_{\text {changing }}=4.07 \times 10^{-3} \mu^{-3 / 11} \mathrm{M}^{-2 / 11} \mathrm{~T}^{-6 / 11} \mathrm{~Hz} \text {. }
$$

This is the frequency at which the system would change by $\Delta f=1 / T$ in the observing time $T$. This is accessible from space, and it shows that Galactic coalescing binary systems can be identified, even if they cannot be followed to coalescence. (This equation is roughiy equivalent to Eq. (35) of 17/.) We will discuss below the likelihood that the Galaxy contains any such systems.

Finally, what is the highest frequency fintoracion of radiation we could expect a system to emit before it is so drastically altered by the close interactions of its components that it becomes difficult to model? A crude estinate, which omits tidal effects, is to take this upper limit to be the point at which the components would be in contact. This limit is determined by the radius of 
the less compact of the two components. For a system with the same parameters as before, and whose less compact star has a radfus $R \times 10 \mathrm{~km}$, this is

$$
f_{\text {interaction }}=1.3 \times 10^{3} \mathrm{M}^{1 / 2} \mathrm{R}^{-3 / 2} \mathrm{~Hz} \text {. }
$$

Clearly, one does not need to worry about interactions for low-frequency observations of systems consisting of neutron stars and/or black holes, until the mass of the block hole exceeds about $10^{*}$ $M_{0}$. Conversely, one can hope to see interaction effects if one observes messive black holes coalescing. I will return to the significance of this below. If the system contains a white dwarf, its evolution will be the same as for more compact objects of the same mass until it reaches a frequency no higher than about $0.2 \mathrm{~Hz}$. Even if this cutoff is too high to be observed from space, one might be able to see tidal effects beginning at lower frequencies. This problem has been discussed in great detail in ref. $17 \%$.

\section{OBSERVABILITY IN VARIOUS DETECTORS}

There are three space-based methods of detecting coalescing binarles that I will consider: Doppler tracking of interplanetary spacecraft (which is already taking place, e.g. $18,9 /$ ), the so-called 'Skyhook' $/ 10 /$, and an interferometric beam detector $/ 11 \%$

Doppler tracking involves looking for the changes caused by gravitational waves in the round-trip signal-transmission time between the Earth and a spacecraft. It is broadband, down to a bit below $10^{-4} \mathrm{~Hz}$, and its past sensitivity to the amplitude $h$ of a gravitational wave has been of the order of $10^{-14}$ for broadband bursts and $3 \times 10^{-1}$ for continuous waves observed over a 4-month period. The planned Galileo misston will be likely to improve this by an order of magnitude, and foreseeable technical improvements could go as $f a r$ as a further factor of 100 . None of the long-lived coalescing binaries (the galactic sources) would be likely to reach this continuous-wave amplitude, unless it were among the Sun's nearest neighbors. For bursts, we must take 1nto account the importance (mentioned above) of matched filtering of the output in improving the signal-to-noise ratio. This will be important to our analysis of the other detectors as well. For a source that is as narrow band as a coelescing binary, the effect of filtering on a signal of amplitude $h$ is to enhance the signal-to-noise ratio to that which a broad-band burst of amplitude h/n would have, where $n$ is the number of cycles of the waveform while it remains in the bandwidth of the detector $11,6 \%$ In our case $n$ is roughly $f \tau$, where $\tau$ is the lifetime given in Eq. (1.2), and $f$ is the lower limit on the freqency of the detector. Putting this together with the maximum amplitude given in Eq. (1.1), and demanding that the interection frequency given in Eq. (2.3) be within the sensitivity range, we see that only coalescing black holes of mass less than $10^{\prime} \mathrm{M}_{0}$ at distances less than 100 Mpc stand a chance of being detected by the Gallleo mission. Such events are not impossible, but must be very rare in such a small fraction of the Hubble volume. However, future technical improvements might put such systems within reach even at interesting distances like 3-10 Gpc. Clearly, there is strong motivation to pursue such improvements.

The Skyhook would be a fairly narrow-band detector, sensitive between $10^{-1}$ and $10^{-2} \mathrm{~Hz}$ to bursts at a level of $h \sim 10^{-16}$ and to continuous waves at $h \sim 10^{-19}$ if 1 t makes a continuous 4-month observing run. This sensitivity, roughly comparable in amplitude to but higher in frequency than the best that can be expected from Doppler tracking, is not likely to be adequate for galactic coalescing binaries, nor would it allow the Skyhook to see massive black holes coalescing in its frequency range unless their masses are below $10 \% \mathrm{M}$ and their distance considerably less than 500 Mpc. Again, the prospects for this seem slim.

A beam in space, however, could have a much better sensitivity: perhaps as low as $3 \times 10^{-25}$ for continuous waves over 4 months, and $10^{-22}$ for bursts, over a bandwidth of $10^{-3}$ to $10^{-1} \mathrm{~Hz}$. This would be more than adequate to detect galactic coalescing binaries and even strong ones in the Andromeda galaxy (M31). It would also see coalescences of binary black holes of mass $10^{4}-10^{\circ} \mathrm{M}_{0}$ essentially anywhere in the observable universe. The beam in space is the instrument with the most promise for returning useful astrophysical information from gravitational wave observations.

\section{EVENT RATES AND COSMOLOGICAL CONSIDERATIONS}

Although we have good reason to suspect that solar-mass coalescing binary systems exist in reasonable numbers, it is very difficult from present astronomical observations to give a firm estimate of their numbers. The first attempt was by Clark, et al $112 \%$ A more up-to-date review of the factors affecting the estimates and their uncertainties is given by Schutz $/ 13 \%$. The conclusion is that binaries consisting of two neutron stars coalesce at the rate of about 1 per galaxy per $10^{\text {s }}$ years, with an uncertainty that is possibly as large as a factor of 100 elther way. Similar rates would apply to binarles consisting of one neutron star and one white dwarf. The rate for twowhite-dwarf binaries has been estimated by Evans, Iben, and Smarr $77 /$ to be perhaps as large as the rate of Type-I supernovae, which may be caused by the coalescence of two white dwarfs. This would suggest a rate of 0.15 per galaxy per year. 
This leads to estimates of the likelihood of observing any given class of systems. For example, if

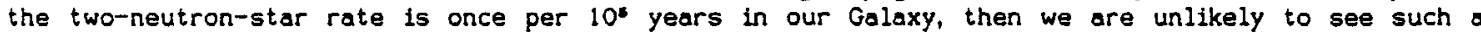
system with an age less than $10^{3}$ years, and therefore with e frequency greater than $4 \times 10^{-3} \mathrm{~Hz}$. But if the rate is 100 times larger, then the highest frequency we might observe is $2 \times 10^{-2} \mathrm{~Hz}_{\text {, }}$

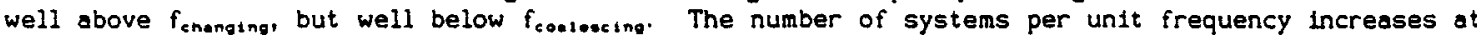
low frequencles as $f^{-111} 17 \%$, so in this optimistic case we might see some 20-30 neutron-star systems above $f_{\text {changing }}$ that could be identifled as coalescing binaries. White dwarf systems would be far commoner. The problem for detection here is the confusion caused by the large number of distant but detectable sources. This affects mainly the low-frequency systems. All systems above $f_{\text {chanoing }}$ ought to stand out from the continuum, even without allowing for the fact that their distinctive frequency change allows them to be flltered from the background $/ 7 \%$. There might be thousands of such systems detectable by a beam in space $17 \%$. These would be indistinguishable from neutron-star systems of similar masses unless tidel effects can be detected; but it may well be that there will be little overlap in the distribution of masses between neutron-star binaries and whitedwarf binaries.

It is equally difficult to give an estimate of the coalescence rate of massive black holes at cosmological distances. It is popular to belleve that active galactic nuclei contain supermassive black holes in the mass range $10^{*}-10^{*} \mathrm{M}_{\theta}$, and there is mounting evidence that ordinary galaxies like our own may contain smaller black holes, perhaps $10^{3}-10^{4} M_{0}$. There are several plausible scenarios for the formation of these holes. One route might be the formation by gas-cloud collapse of black holes of 1000 solar masses, followed by the hterarchical coalescence of larger and larger black holes. If this were the case, then the formation of a $10^{\prime} \mathrm{M}_{0}$ hole would involve a large number of observable coalescences, and would give event rates of several per year, even if only 1 galaxy in 1000 has a supermassive black hole in it. This is the most optimistic scenarlo, of course, and it may be that only gravitational wave observations from space will be able to shed light on the question of the formation of these giant black holes.

When studying coalescing binary systems that are at cosmological distances, it is important to take Into account the effects of the redshift $z$. It can be shown that this is very simple: Eqs. (1.1) \& (1.2) stfll apply, but the 'observed' masses $\mu$ and $M$ are $(1+z)$ times the true values of the masses, as measured near the source, and the distance $r$ is the luminosity distance. The frequency $f$ in the equations is the observed frequency, which is also redshifted from the original frequency. If a given system is taken to larger and larger redshifts, and if the system can be followed all the way to coalescence, then the signal-to-noise ratio will depend on redshift to lowest nontrivial order as

$$
S / N \times z^{-1}\left[1+\left(\frac{2+3 q_{0}}{6}\right) z+\ldots\right] .
$$

Here $q_{0}$ is the usual deceleration parameter $/ 14 \%$. The $1 / 2$ dependence is just what one expects, but the effect of going to higher redshifts is to increase the signal-to-noise ratio above what one would expect: in this sense, coalescing binaries are easier to detect at larger redshifts.

\section{WHAT WE WOULD LEARN FROM COALESCING BINARIES}

From our discussion above of the sorts of systems that would be candidates for observation, it is clear that very fundamental astrophysical information is available in these waves. Let us start with the smaller, local systems first.

An important unknown is the number and distribution (in age and frequency) of coalescing binary systems in our Galaxy. By the time space observations with sufficient sensitivity take place, we should already have a good idea from ground-based detections what the typical neutron-star coalescence rate should be, averaged over some $10^{6}$ galaxies or more. But it is possible that several distinct populations contribute to the rate: old systems (Populations II and III) that formed with or soon after the Galaxy and whose binaries are gradually decaying, and new systems (Population I) that are still forming precursor systems. By observing the spatial distribution of coalescing binary systems in our Galaxy, these two populations could be distinguished, and this would in turn tell us much about the differences between star formation now and in the remote past. (This will not be easy to do with only one detector in space, however.) Among the coalescing systems might be a few percent that consist of a neutron star and a black hole, and it would be interesting to see these.

Seeing white-dwarf binaries would be equally interesting. If their numbers correspond to a birthrate equal to the rate of supernovae of Type I, this would be strong evidence for the idea that they end as such supernovae. Observations of tidal effects in white dwarf binaries would also give considerable information about pre-supernova systems and about accretion and mass exchange in interacting binaries.

The cosmological systems would of course be even more drematic. Not only would the1r detection shed light on the nature and formation of active galactic nuclei and quasi-stellar objects, but they wold also orovide a stringent test of general relativity: once the collapse was followed far 


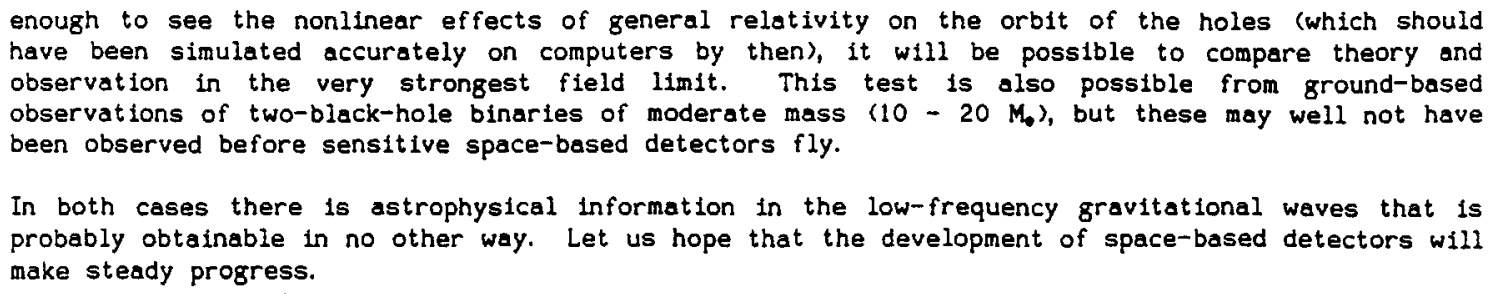

In both cases there is astrophysical information in the low-frequency gravitational waves that is probably obtainable in no other way. Let us hope that the development of space-based detectors will make steady progress.

\section{REFERENCES}

1. K.S. Thorne, in: 300 Years of Gravitation, eds. S.W. Hawking and W. Israel, Cambridge University Press, Cambridge, 1987.

2. J.P.A. Clark and D.M. Eardley, Astrophys. J., 215, 315 (1977).

3. A. Krolak and B.F. Schutz, Gen. Rel. Gravit., 19, 1163 (1987).

4. B.F. Schutz, Nature, 323, 310 (1986),

5. B.F. Schutz, In: Gravitational Collapse and Relativity, eds. H. Sato and T. Nakamura, World Scient1fic, Singapore, 1986.

6. B.F. Schutz, ed., Gravitational Wave Dato Analysis, Reidel, Dordrecht, 1988.

7. C.R. Evans, I. Iben, Jr., and L. Smarr, Degenerate Dwarf Binaries as Promising Detectable Sources of Gravitational Radiation, California Institute of Technology preprint GRP-123, March 1987.

8. J.W. Armstrong, F.B. Estabrook, and H.D. Wahlquist, Astrophys. J., 318, 536 (1987).

9. R.W. Hellings, P.S. Callahan, J.D. Anderson, and A.T. Moffet, Phys. Rev., D23, 844 (1981).

10. V.B. Braginsky and K.S. Thorne, Nature, 316, 610 (1985).

11. J.E. Faller, P.L. Bender, J.L. Hall, D. Hils, R.T. Stebbins, and M.A. Vincent, this volume.

12. J.P.A. Clark, E.P.J. van den Heuvel, and W. Sutantyo, Astron. Astrophys., 72, 120 (1979).

13. B.F. Schutz, in: /6/,

14. B.F. Schutz, A First Course in General Relativity, Cambridge University Press, Cambridge, 1985. 